\title{
Prediction of protein structural classes using hybrid properties
}

\author{
Wenjin Li · Kao Lin · Kaiyan Feng · Yudong Cai
}

Received: 5 August 2008 / Accepted: 25 September 2008 / Published online: 25 October 2008

(c) Springer Science+Business Media B.V. 2008

\begin{abstract}
In this paper, amino acid compositions are combined with some protein sequence properties (physiochemical properties) to predict protein structural classes. We are able to predict protein structural classes using a mathematical model that combines the nearest neighbor algorithm (NNA), mRMR (minimum redundancy, maximum relevance), and feature forward searching strategy. Jackknife cross-validation is used to evaluate the prediction accuracy. As a result, the prediction success rate improves to $68.8 \%$, which is better than the $62.2 \%$ obtained when using only amino acid compositions. Therefore, we conclude that the physiochemical properties are factors that contribute to the protein folding phenomena and the most contributing features are found to be the amino acid composition. We expect that prediction accuracy will improve further as more sequence information comes to light. A web server for predicting the protein structural classes is available at http:// app3.biosino.org:8080/liwenjin/index.jsp.
\end{abstract}

Keywords Protein structural class - Nearest neighbor algorithm · mRMR (Minimum Redundancy, Maximum Relevance) - Physiochemical properties · Amino acid compositions

Electronic supplementary material The online version of this article (doi:10.1007/s11030-008-9093-9) contains supplementary material, which is available to authorized users.

W. Li · K. Lin · Y. Cai $(\varangle)$

CAS-MPG Partner Institute for Computational Biology, Shanghai Institutes for Biological Sciences, Chinese Academy of Sciences, Shanghai, China

e-mail: cyd@picb.ac.cn

\section{K. Feng}

Division of Imaging Science \& Biomedical Engineering, The University of Manchester, Room G424, Stopford Building, Manchester, M13 9PT, UK

\section{Introduction}

Three-dimensional (3-D) structures of proteins are closely related to their primary structure - their amino acid sequence. For many years scientists have tried to develop a prediction model to correlate amino acid sequences to the protein structures. Although early prediction studies only used amino acid compositions without amino acid sequence data [1-7], one study was capable of predicting a 3-D protein structure with $84 \%$ accuracy [8]. With the realization that amino acid sequences contribute factors to increase the accuracy of protein folding predictions, many researchers added more information related to the sequence, such as the pseudo amino acid compositions which involve not only amino acid compositions but also the sequence-order and length information [9], hydropobicity, polarity and distribution of certain amino acids [10-13] to enhance the prediction capability. Recently, Ding [14] employed eight physiochemical features to construct pseudo amino acid compositions and managed to gain 92.6\% prediction accuracy using dual-layer fuzzy support vector machine (FSVM) network. Ding's work only covered 204 proteins and four structural classes: all $\alpha$ all $-\beta, \alpha / \beta$ and $\alpha+\beta$.

This paper does not aim for an increase in the prediction rate. Instead, we are investigating whether combining the physiochemical properties and amino acid compositions are better than using amino acid compositions alone in predicting the protein structural classes. We will also test the hypothesis that the physiochemical properties, derived from the amino acid sequence arrangement, contribute more to the prediction of protein structural classes than using the amino acid compositions alone. We will demonstrate that, by combining the mRMR (Minimum Redundancy, Maximum Relevance) [15] and forward feature selections, we are able to first optimize the prediction model and second increase the efficiency of 
building the prediction model. Instead of the traditional four structural classes we extend them to seven structural classes, which include all- $\alpha$, all- $\beta, \alpha / \beta, \alpha+\beta$, multi-domain proteins, membrane and cell surface proteins, small proteins. We also use a fairly large data set with 12520 proteins to minimize the defect of getting a high correct prediction rate by chance. Nearest neighbor algorithm (NNA) [16] is used to predict which structural class a query protein should be placed.

\section{Materials and methods}

\section{Dataset}

According to the SCOP ("Structural Classification of Proteins") [17-19], proteins belong to seven structural classes: all alpha proteins (all- $\alpha$ ), all beta proteins (all- $\beta$ ), alpha and beta proteins $(\alpha / \beta)$, alpha and beta proteins $(\alpha+\beta)$, multidomain proteins $(\gamma)$, membrane and cell surface proteins $(\delta)$, and small proteins $(\zeta)$. The dataset we are using, including 2299 all- $\alpha, 3334$ all- $\beta, 3086 \alpha / \beta, 2870 \alpha+\beta, 224 \gamma, 227 \delta$, and $984 \zeta$, is released by ASTRAL (release 1.71, 2007, http:// astral.berkeley.edu/) [20-22]. After removing the proteins whose sequence containing unnatural amino acids, we get the refined dataset with 2270 all- $\alpha, 3199$ all- $\beta, 2842 \alpha / \beta$, $2812 \alpha+\beta, 213 \gamma, 222 \delta$, and $962 \zeta$-totally 12520 proteins (refer to Table 1 and supplemental material 1).

\section{Combined protein sequence descriptors}

Each protein is represented as a 111-dimensional vector which consists of 20 amino acid compositions and 91 physiochemical features. The physiochemical features include the properties of: hydrophobicity, normalized Van Der Waals volume, polarity, polarizability and solvent accessibility.

(1) Amino acid compositions: the percentage of each of the normal 20 amino acids occurring in the whole sequence.

(2) Hydrophobicity, normalized Van Der Waals volume, polarity and polarizability: global description of the amino

Table 1 Description of the dataset

The original dataset is from ASTRAL SCOP release 1.71 and the refined dataset used in this study

\begin{tabular}{lrc}
\hline Class & $\begin{array}{l}\text { Original } \\
\text { dataset }\end{array}$ & $\begin{array}{l}\text { Dataset } \\
\text { after } \\
\text { refinement }\end{array}$ \\
\hline All- $\alpha$ & 2299 & 2270 \\
All- $\beta$ & 3334 & 3199 \\
$\alpha / \beta$ & 3086 & 2842 \\
$\alpha+\beta$ & 2870 & 2812 \\
$\gamma$ & 224 & 213 \\
$\delta$ & 227 & 222 \\
$\zeta$ & 984 & 962 \\
Overall & 13006 & 12520 \\
\hline
\end{tabular}

acid sequence can be used to obtain 21 features for each of these properties [10,23].

The method for obtaining global properties such as hydrophobicity are as follows: First, each amino acid is classified into three categories - polar, neutral and hydrophobic amino acid [23]. For a given protein sequence, the polar amino acids, R,K,E,D,Q,N, are substituted by character $P$, the neutral amino acids, G,A,S,T,P,H,Y, are substituted by character $\mathrm{N}$, and the hydrophobic amino acids, C,V,L,I,M,F,W, are substituted by character $\mathrm{H}$. Thus each protein property sequence is a sequence of $\mathrm{P}, \mathrm{N}$, and $\mathrm{H}$, instead of amino acids. Then, composition (C) is taken as the percentage of $\mathrm{P}, \mathrm{N}$ or $\mathrm{H}$; transition $(\mathrm{T})$ is defined as the changing frequency between two different properties (such as the transition from $\mathrm{P}$ to $\mathrm{N}$, or $\mathrm{P}$ to $\mathrm{H}$, or $\mathrm{H}$ to $\mathrm{N}$ ); distribution (D) is defined as how much of the protein sequence is needed to contain $25 \%$, $50 \%, 75 \%$ and $100 \%$ of the Ps, Ns and Hs, respectively. An example below shows how these percentages and how all 21 features are obtained.

Suppose a property sequence under examine contains 9 Ps, 16 Ns and $11 \mathrm{Hs}$ (totally 36), as showed in Fig. 1. The compositions (C) for $\mathrm{P}, \mathrm{N}$ and $\mathrm{H}$ are $(9 / 36) * 100 \%=25 \%$, $(16 / 36) * 100 \%=44.4 \%$ and $(11 / 36) * 100 \%=30.6 \%$, respectively. The numbers of transitions between $\mathrm{P}$ and $\mathrm{N}$, between $\mathrm{P}$ and $\mathrm{H}$ and between $\mathrm{N}$ and $\mathrm{H}$ are 9, 7, and 10, respectively. Therefore, the three transitions ( $\mathrm{T}$ ) between $\mathrm{P}, \mathrm{N}$ and $\mathrm{H}$ are $(9 / 36) * 100 \%=25 \%,(7 / 36) * 100 \%=19.4 \%$ and $(10 / 36) * 100 \%=27.8 \%$, respectively. In this case, we search $\mathrm{P}$ in the property sequence from $\mathrm{N}$ terminal to $\mathrm{C}$ terminal. The first $\mathrm{P}$ appears in the 4 th of the property sequence, i.e. the length of the first segment is 4 . Therefore, the first distribution (D) value for $\mathrm{P}$ is $(4 / 36) * 100 \%=11.1 \%$. When the $25 \%$ of Ps are included, the length of the segment is 5 . The second distribution (D) value for $\mathrm{P}$ is thus $(5 / 36) * 100 \%=$ $13.9 \%$, and so forth. The third, forth and fifth distribution (D) values are $(13 / 36) * 100 \%=36.1 \%,(22 / 36) * 100 \%=$ $61.1 \%$ and $(34 / 36) * 100 \%=94.4 \%$, respectively. Similarly, the five distribution values for $\mathrm{N}$ are $2.8 \%, 27.8 \%$, $47.2 \%, 75 \%, 97.2 \%$, and the five distribution values for $\mathrm{H}$ are $5.6 \%, 8.3 \%, 41.7 \%, 80.6 \%, 100 \%$. Therefore, the property sequence derived from hydrophobicity property can produce 21 features: 3 for composition $\mathrm{C}(0.25,0.444,0.306)$, 3 for transition $\mathrm{T}(0.25,0.194,0.278)$ and 15 for distribution $\mathrm{D}(0.111,0.139,0.361,0.611,0.944,0.028,0.278,0.472$, $0.75,0.972,0.056,0.083,0.417,0.806,1)$.

Using the same way described above, the 21 features can be obtained from each of the other three physiochemical properties: normalized Van Der Waals volume, polarity and polarizability, respectively.

(3) Solvent accessibility: residues of a protein can be divided into two group (buried and exposed). A residue is considered as exposed if the percentage of the exposed surface is larger than $20 \%$. The solvent accessibility of a protein 


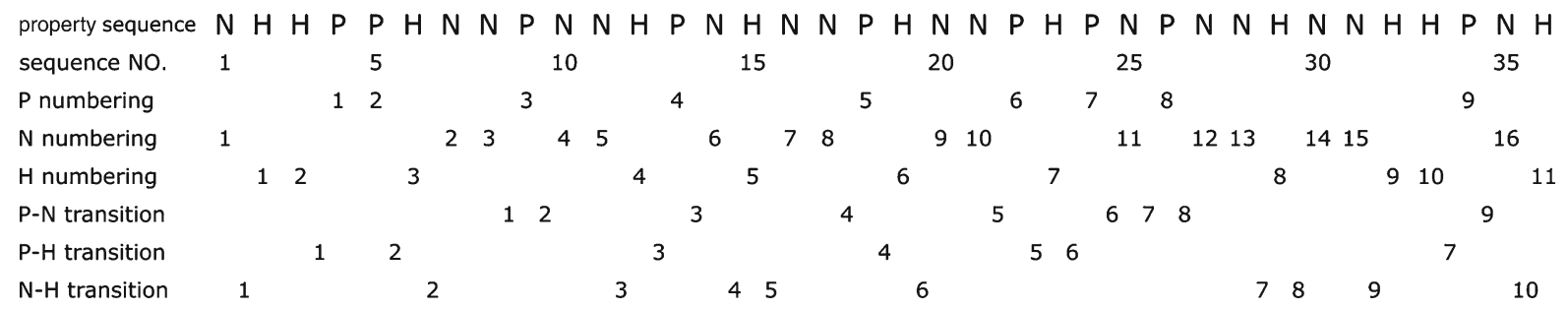

Fig. 1 Analyzing property sequence derived from hydrophobicity property

is obtained by PredAcc [24] in this study. We denote the exposed residues and exposed residues with gamma risks as $\mathrm{E}$, and the hidden residues and hidden residues with gamma risks as $\mathrm{H}$, from which we can obtain a property sequence with only $\mathrm{E}$ and $\mathrm{H}$. Only composition for $\mathrm{H}$, transition between $\mathrm{H}$ and $\mathrm{E}$, and five distributions for $\mathrm{H}$ are considered as useful features (totally 7) to avoid feature redundancy [10].

In conclusion, the total number of features is $20+21 * 4+$ $7=111$ (see Table 2). Each protein sequence is transferred into 111-dimensional vector (see supplemental materials 2), which needs to be normalized by the following equations:

$\mu_{j}=\sum_{i=1}^{n} v_{i j} / n$

$S_{j}=\sqrt{\sum_{i=1}^{n}\left(v_{i j}-\mu_{j}\right)^{2} /(n-1)}$

$V_{i j}=\frac{v_{i j}-\mu_{j}}{S_{j}}$

where $v_{i j}$ is the value of the $j$ th feature in the $i$ th protein, $n$ is the total number of proteins, $S_{j}$ is the standard deviation of the $j$ th feature, and $V_{i j}$ is the normalization value of $j$ th feature in the $i$ th protein.

Minimum redundancy-maximum relevance (mRMR) $[15,25]$

Feature selection can very effectively reduce the feature dimensions, improve a learning machine's generalization,

Table 2 Feature distribution

\begin{tabular}{|c|c|c|c|c|}
\hline \multirow[t]{2}{*}{$\begin{array}{l}\text { Sequence } \\
\text { properties }\end{array}$} & \multicolumn{3}{|c|}{$\begin{array}{l}\text { The number of features } \\
\text { in certain property }\end{array}$} & \\
\hline & $\mathrm{C}$ & $\mathrm{T}$ & $\mathrm{D}$ & \\
\hline Amino acid composition & \multicolumn{3}{|c|}{20} & 20 \\
\hline Hydrophobicity & 3 & 3 & 15 & 2 \\
\hline Normalized Van Der Waal volume & 3 & 3 & 15 & 21 \\
\hline Polarity & 3 & 3 & 15 & 21 \\
\hline Polarizability & 3 & 3 & 15 & 21 \\
\hline Solvent accessibility & 1 & 1 & 5 & 7 \\
\hline
\end{tabular}

facilitating the data mining task. We choose mRMR feature selection algorithm because it is able to balance the minimum redundancy and the maximum relevance in a simple and elegant mathematical way. The maximum relevance part looks for features that contribute most to the classification, and the minimum redundancy part tries to exclude the features whose prediction capability has been included by the already selected features. The algorithm is described briefly as below:

Given a dataset $\Omega$ including all features, we are seeking a subset $S$ of the features to satisfy both the minimum redundancy and the maximum relevance conditions.

Firstly, the mutual information $I$ of two variables $\mathrm{x}$ and $\mathrm{y}$ is defined as:

$I(x, y)=\sum_{i, j} P\left(x_{i}, y_{j}\right) \log \frac{P\left(x_{i}, y_{j}\right)}{P\left(x_{i}\right) P\left(y_{j}\right)}$

where $P\left(x_{i}, y_{j}\right)$ is the joint probabilistic distribution of $x_{i}$ and $y_{j} ; P\left(x_{i}\right)$ and $P\left(y_{j}\right)$ are the marginal probabilities of $x_{i}$ and $y_{j}$, respectively.

The minimum redundancy is calculated as:

$\min _{S \subseteq \Omega} W_{I}, W_{I}=\frac{1}{|S|^{2}} \sum_{i, j \in S} I\left(x_{i}, x_{j}\right)$

where $|S|$ is the number of features in $S, W_{I}$ is the minimum redundancy value.

For the targeted classes $h=\left\{h_{1}, h_{2}, \ldots, h_{k}\right\}$ (i.e. the class variable), the relevance between feature $i$ and the targeted class variable $h$ can be quantified by the mutual information $I\left(h, x_{i}\right)$ between $h$ and the feature variable $x_{i}$. The maximum relevance can be obtained by the following formula:

$\max _{S \subseteq \Omega} V_{I}, V_{I}=\frac{1}{|S|} \sum_{i \in S} I\left(h, x_{i}\right)$

where $V_{I}$ is the maximum relevance value.

In order to optimize both Eqs. 5 and 6, mRMR is accomplished by the following steps:

(1) Select a single most relevant feature according to Eq. 6 , i.e. select feature $i$ such that $I\left(h, x_{i}\right)$ is higher than other features.

$$
\max _{i \in \Omega} I\left(x_{i}, h\right)
$$


Fig. 2 The accuracy curves produced by the mRMR features (Black Square) and the forward feature searching method from the 19th feature (Grey Triangle)

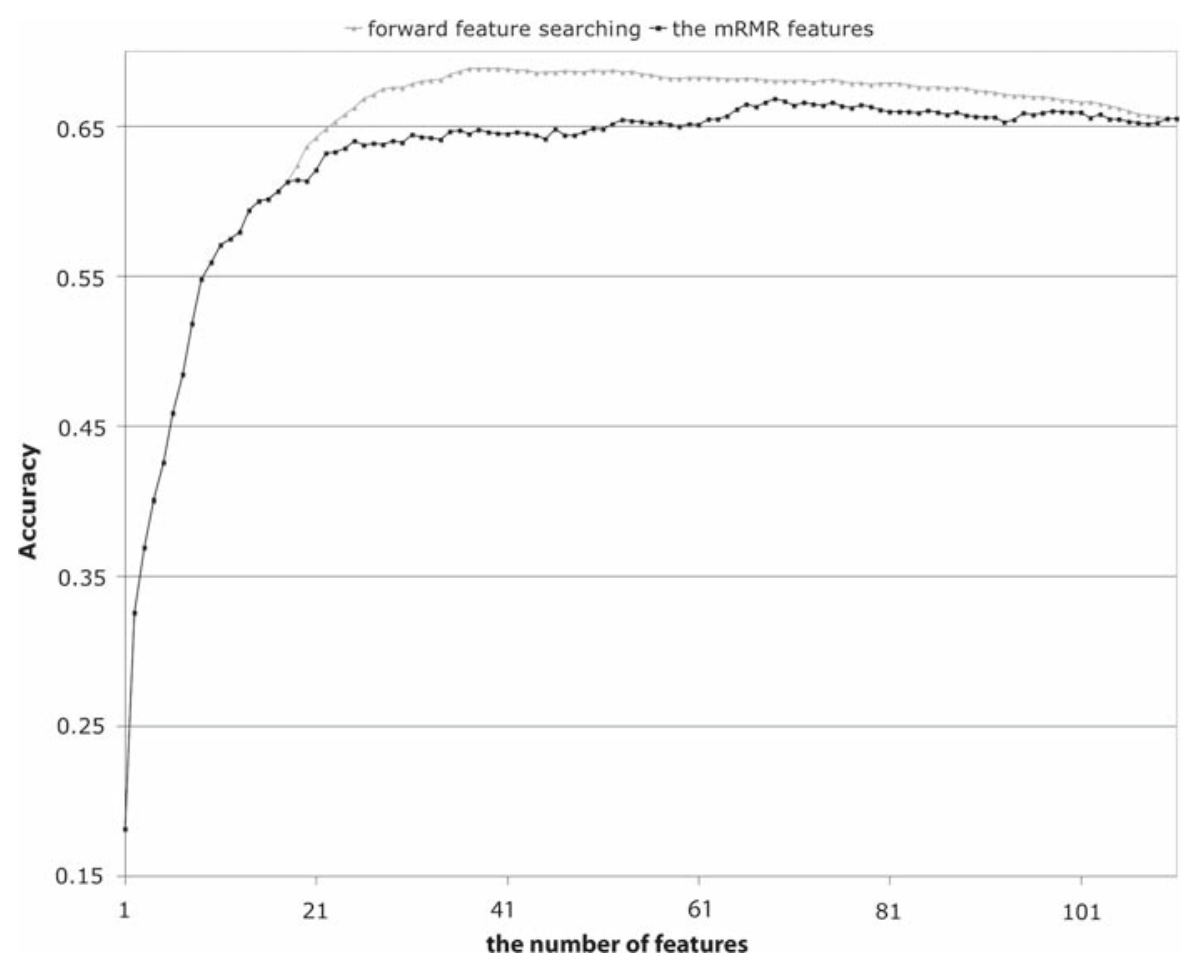

condition:

$\max _{i \in \Omega_{S}} A(S \cup\{i\})$

where $A(S \cup\{i\})$ means the prediction accuracy obtained by the prediction model evaluated by an evaluation method such as the jackknife test.

\section{Results and discussion}

As described in Sect. "Combined protein sequence descriptors", each protein is represented by a 111-dimentional space. Thus the 12520 proteins can be expressed by a $12520 * 111$ matrix (shown in supplemental material 2). Using mRMR method (see Sect. "Minimum redundancy-maximum relevance"), we obtain two feature lists (see supplemental material 3): the first list, named as maxRel features, showing the features in maximum relevance order, and the second list, named as mRMR features, showing the features selected by Eqs. 7 and 8 in a selection order. In order to find out how many foremost features in the mRMR feature list should be included for the prediction model, we add one feature at a time from the list in order and obtain the prediction accuracy for the selected features using jackknife test. In this study, we choose to run all the features in the list and gain the optimized feature subset that achieves the highest prediction accuracy. A scheme is designed to adjust between finding global prediction accuracy and local prediction accuracy if one encounters a large feature set. As a result, the highest accuracy is found to be $66.8 \%$ and it takes place when the 
Table 3 The order and name of mRMR features with the prediction accuracies evaluated by jackknife cross-validation test

\begin{tabular}{|c|c|c|c|c|c|}
\hline 1 & AA_composition_C & 0.18131 & 57 & Hydrophobicity_transition_PH & 0.652476 \\
\hline 2 & Solvent_composition_H & 0.325799 & 58 & Polarity_distribution_N-1.0 & 0.650799 \\
\hline 3 & AA_composition_L & 0.369329 & 59 & Polarizability_distribution_N-1.0 & 0.649361 \\
\hline 4 & Polarity_composition_H & 0.400639 & 60 & Hydrophobicity_distribution_P-0.75 & 0.651118 \\
\hline 5 & AA_composition_V & 0.425479 & 61 & Polarity_distribution_H- 0.0 & 0.650639 \\
\hline 6 & AA_composition_T & 0.458626 & 62 & Hydrophobicity_transition_PN & 0.654712 \\
\hline 7 & VanDerWaal_composition_N & 0.483946 & 63 & Polarity_composition_N & 0.654633 \\
\hline 8 & AA composition A & 0.518211 & 64 & AA composition $\mathrm{Y}$ & 0.656629 \\
\hline 9 & AA_composition_G & 0.547764 & 65 & Polarity_distribution_P-0.25 & 0.661022 \\
\hline 10 & AA_composition_S & 0.558866 & 66 & AA_composition_R & 0.664297 \\
\hline 11 & AA_composition_M & 0.570607 & 67 & Hydrophobicity_distribution_H-1.0 & 0.66262 \\
\hline 12 & VanDerWaal_distribution_N-0.25 & 0.574601 & 68 & AA_composition_K & 0.665575 \\
\hline 13 & Solvent_distribution_H-0.0 & 0.579073 & 69 & Hydrophobicity_distribution_H-0.75 & 0.668211 \\
\hline 14 & AA_composition_W & 0.59369 & 70 & Polarity_distribution_H-1.0 & 0.666613 \\
\hline 15 & VanDerWaal_composition_P & 0.6 & 71 & Hydrophobicity_distribution_N-1.0 & 0.663578 \\
\hline 16 & Solvent_distribution_H- $0 . \overline{7} 5$ & 0.601118 & 72 & Polarity_transition_PN & 0.665575 \\
\hline 17 & VanDerWaal_distribution_H-0.0 & 0.606629 & 73 & Polarizability_distribution_N-0.5 & 0.664457 \\
\hline 18 & AA_composition_E & 0.612859 & 74 & AA_composition_F & 0.663419 \\
\hline 19 & VanDerWaal_distribution_N-1.0 & 0.613898 & 75 & Polarity_distribution_N-0.75 & 0.665575 \\
\hline 20 & Solvent_transition_HE & 0.613099 & 76 & Hydrophobicity_distribution_H-0.25 & 0.663259 \\
\hline 21 & Solvent_distribution_H-0.25 & 0.620687 & 77 & VanDerWaal_transition_PN & 0.661901 \\
\hline 22 & AA_composition_P & 0.631629 & 78 & Polarity_distribution_H-0.5 & 0.664137 \\
\hline 23 & VanDerWaal_distribution_N-0.0 & 0.632428 & 79 & AA_composition_Q & 0.66278 \\
\hline 24 & VanDerWaal_distribution_N-0.5 & 0.635064 & 80 & Polarity_transition_NH & 0.660863 \\
\hline 25 & Hydrophobicity composition $\mathrm{H}$ & 0.640096 & 81 & VanDerWaal distribution P-0.0 & 0.659505 \\
\hline 26 & Hydrophobicity_distribution_- -0.0 & 0.637141 & 82 & Polarizability_distribution_P-0.25 & 0.659505 \\
\hline 27 & Polarity_distribution_P- 0.0 & 0.638578 & 83 & Polarizability_composition_P & 0.659425 \\
\hline 28 & Hydrophobicity_composition_P & 0.63746 & 84 & Hydrophobicity_distribution_P-1.0 & 0.658786 \\
\hline 29 & AA_composition_I & 0.640096 & 85 & Polarity_distribution_H- 0.25 & 0.660304 \\
\hline 30 & Solvent_distribution_H-0.5 & 0.638658 & 86 & Polarity_distribution_P-0.5 & 0.658866 \\
\hline 31 & VanDerWaal_transition_NH & 0.644089 & 87 & Polarizability_composition_N & 0.657508 \\
\hline 32 & VanDerWaal_distribution_N-0.75 & 0.642572 & 88 & Polarity_distribution_N-0.5 & 0.658946 \\
\hline 33 & Polarizability_distribution_P-1.0 & 0.642013 & 89 & Polarity_transition_PH & 0.656869 \\
\hline 34 & VanDerWaal_distribution_H-1.0 & 0.640974 & 90 & Polarity_distribution_H-0.75 & 0.65599 \\
\hline 35 & AA_composition_D & 0.646086 & 91 & VanDerWaal_distribution_H-0.25 & 0.655911 \\
\hline 36 & Polarizability_distribution_P-0.0 & 0.646965 & 92 & Polarizability_distribution_N-0.75 & 0.655751 \\
\hline 37 & Polarizability_distribution_N-0.0 & 0.644728 & 93 & Polarity_distribution_N-0.25 & 0.652476 \\
\hline 38 & Hydrophobicity_composition_N & 0.647684 & 94 & Hydrophobicity_distribution_N-0.75 & 0.653994 \\
\hline 39 & Polarizability_distribution_H- 0.0 & 0.645767 & 95 & AA_composition_N & 0.658626 \\
\hline 40 & Solvent_distribution_H-1.0 & 0.644808 & 96 & VanDerWaal_distribution_H-0.75 & 0.657268 \\
\hline 41 & VanDerWaal_transition_PH & 0.644728 & 97 & Polarizability_composition_H & 0.658466 \\
\hline 42 & Hydrophobicity_distribution_P-0.0 & 0.645927 & 98 & Polarizability_transition_P $\bar{H}$ & 0.659744 \\
\hline 43 & Polarizability_distribution_N-0.25 & 0.644968 & 99 & VanDerWaal_distribution_P-0.25 & 0.659665 \\
\hline 44 & Hydrophobicity_transition_NH & 0.64369 & 100 & Hydrophobicity_distribution_P-0.5 & 0.658946 \\
\hline 45 & Hydrophobicity_distribution_H-0.0 & 0.641134 & 101 & Polarizability_transition_PN & 0.659185 \\
\hline 46 & Polarity_distribution_P-0.75 & 0.647923 & 102 & VanDerWaal_distribution_P-1.0 & 0.655351 \\
\hline 47 & Polarity_distribution_N-0.0 & 0.64377 & 103 & Hydrophobicity_distribution_N-0.5 & 0.657827 \\
\hline 48 & Polarizability_distribution_P-0.75 & 0.64361 & 104 & VanDerWaal_distribution_P-0.75 & 0.654712 \\
\hline 49 & Hydrophobicity_distribution_P-0.25 & 0.645927 & 105 & VanDerWaal_distribution_H-0.5 & 0.654393 \\
\hline 50 & Polarity_composition_P & 0.648163 & 106 & VanDerWaal_distribution_P-0.5 & 0.653035 \\
\hline 51 & Polarity_distribution_P-1.0 & 0.647843 & 107 & Hydrophobicity_distribution_N-0.25 & 0.651997 \\
\hline 52 & AA_composition_H & 0.651278 & 108 & Polarizability_distribution_H- 0.75 & 0.651358 \\
\hline 53 & Polarizability_distribution_H- 0.25 & 0.654073 & 109 & VanDerWaal_composition_H & 0.651837 \\
\hline 54 & Hydrophobicity_distribution_H-0.5 & 0.653195 & 110 & Polarizability_transition_NH & 0.654872 \\
\hline 55 & Polarizability_distribution_H-1.0 & 0.652955 & 111 & Polarizability_distribution_H- 0.5 & 0.654952 \\
\hline
\end{tabular}

AA_composition_C means the amino acid composition of amino acid C, solvent_composition_H is the H compositon obtained from the solvent accessibility of proteins, and so forth. The highest accuracy is $66.8 \%$, which takes place when the 69th feature is added in 
Fig. 3 The accuracy curve using only amino acid compositions: one feature is added each time in the order listed in the mRMR features

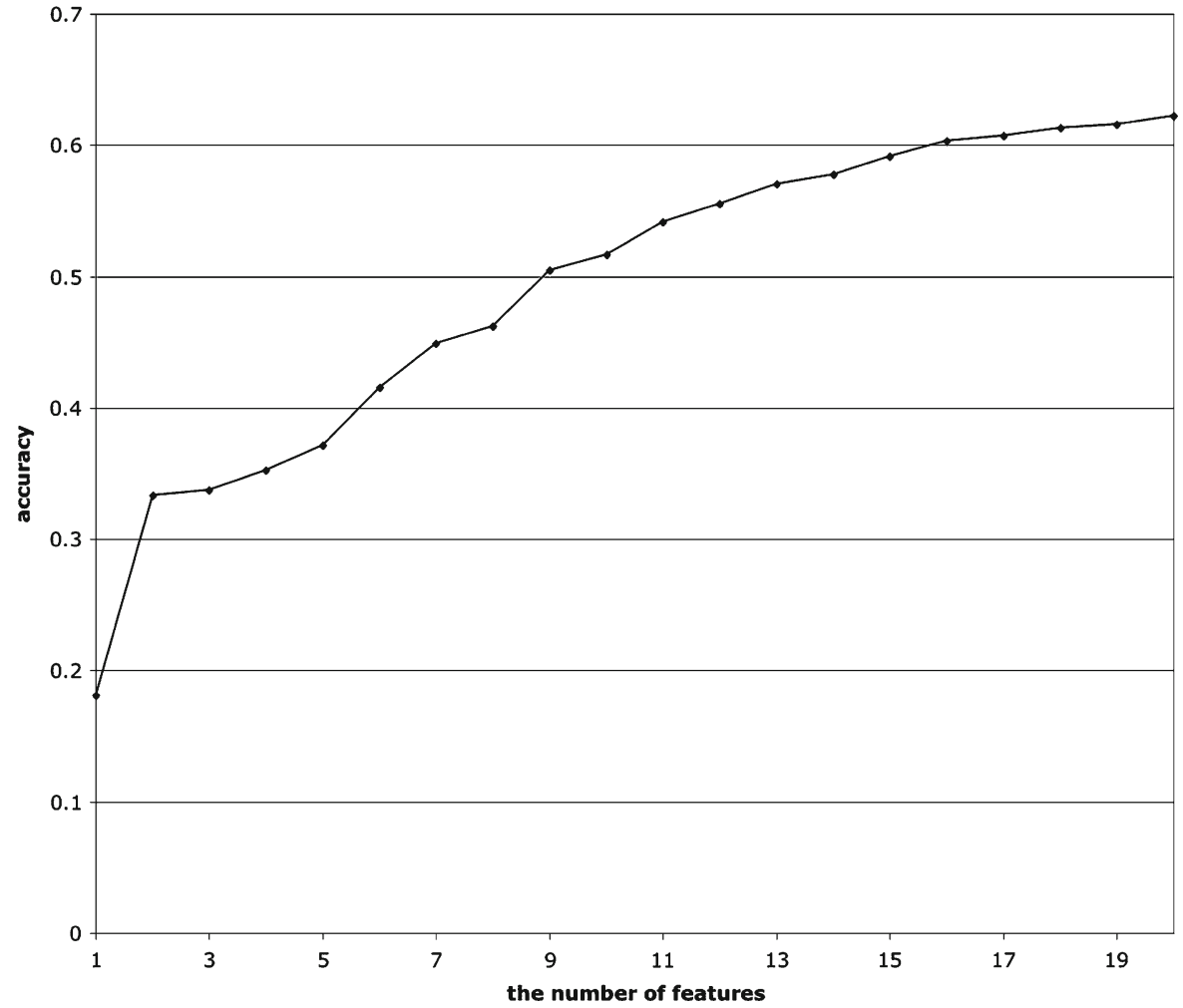

69th feature is added (see Fig. 2, Table 3). We also carry out a prediction using pure amino acid compositions in the same way as the 111-dimensional features. The highest accuracy is $62.2 \%$ (see Fig. 3) when all 20 amino acid compositions are included. We conclude that physiochemical properties do provide some extra prediction capability to the original amino acid compositions.

As we mentioned above, the mRMR method does not involve the mathematical prediction model. An issue is then brought forth how to combine mRMR method with forward feature searching method. The mRMR method is good at fast computation while the forward feature searching method is more accurate and often provides better selection results. The mRMR method can provide an initial optimized feature selection quickly, and the time for processing the forward feature selection is polynomial to the size of a feature set. In this study, we choose to select the foremost features using mRMR method and the rest features using forward feature selection method. Our choice may not be optimized as there are more ways to combine them to balance between speed and accuracy. Our method best compares the forward feature selection and the mRMR in parallel curves as described below. We choose the first 18 features of mRMR features (see Table 3) as the initial feature subset, which has reduced some computation burden left for the forward feature selection method. The first $18 \mathrm{mRMR}$ features are chosen mainly because they continuously give an ascending accuracy curve until it achieves an accuracy of $61.3 \%$. Then, we search the rest of the features using forward feature searching strategy, and gain an accuracy of $68.8 \%$ when 37 features are selected (see Fig. 2; Table 4). If more than 37 features are selected, the prediction model suffers more from the overfitting problem and the prediction accuracy deteriorates as the number of features increases. By comparing the two curves after the 18 features are selected in the $x$ axis in Fig. 2, the forward feature selection method does provide better feature selection results as the compensation of more computation. Could one divide the features from mRMR method into several fragments and use the forward feature selection method in each of those fragments? This aspect will be investigated in the future.

Table 5 lists the 22 foremost features taken from the maxRel features (i.e. the maximum relevant part in the mRMR feature selection). The foremost $18 \mathrm{mRMR}$ features can all be found in these 22 maxRel features. This tells that the maximum relevance is weighted strongly towards building the mRMR features. One could introduce a weighting parameter in Eqs. 8 or 9 to adjust the balance between maximum relevance and the minimum redundancy. In this study, the maxRel features were weighted strongly. We will leave the study of the balance between maximum relevance and the minimum redundancy in the future.

The arrangement of the amino acids in the protein impart more information than the amino acid composition alone. Therefore, we hypothesize that the physiochemical properties derived from the sequence arrangement should contribute more to the prediction of protein structural classes. Along with testing the hypothesis, we have also done the feature 
Table 4 The order and names of the features selected by the forward feature searching method with the prediction accuracies evaluated by the jackknife cross-validation

\begin{tabular}{|c|c|c|c|c|c|}
\hline 1 & AA_composition_C & 0.18131 & 57 & Hydrophobicity_composition_H & 0.682748 \\
\hline 2 & Solvent_composition_H & 0.325799 & 58 & VanDerWaal_distribution_N-0.5 & 0.681789 \\
\hline 3 & AA_composition_L & 0.369329 & 59 & Hydrophobicity_distribution_H-0.25 & 0.681949 \\
\hline 4 & Polarity_composition_H & 0.400639 & 60 & Hydrophobicity_distribution_N-0.5 & 0.682189 \\
\hline 5 & AA_composition_V & 0.425479 & 61 & Polarity_distribution_H- 0.25 & 0.682029 \\
\hline 6 & AA_composition_T & 0.458626 & 62 & Polarity_distribution_P-0.25 & 0.682109 \\
\hline 7 & VanDerWaal_composition_N & 0.483946 & 63 & Polarizability_distribution_P-0.25 & 0.681949 \\
\hline 8 & AA_composition_A & 0.518211 & 64 & Polarity_distribution_P- 0.5 & 0.68131 \\
\hline 9 & AA_composition_G & 0.547764 & 65 & Polarity_distribution_P-1.0 & 0.68123 \\
\hline 10 & AA_composition_S & 0.558866 & 66 & AA_composition_I & 0.681629 \\
\hline 11 & AA_composition_M & 0.570607 & 67 & Hydrophobicity_distribution_N-0.75 & 0.68123 \\
\hline 12 & VanDerWaal_distribution_N-0.25 & 0.574601 & 68 & Polarity_distribution_H-1.0 & 0.680431 \\
\hline 13 & Solvent_distribution_H-O.0 & 0.579073 & 69 & VanDerWaal_transition_NH & 0.680272 \\
\hline 14 & AA_composition_W & 0.59369 & 70 & Polarizability_distribution_N-0.5 & 0.680032 \\
\hline 15 & VanDerWaal_composition_P & 0.6 & 71 & Hydrophobicity_distribution_H-1.0 & 0.680351 \\
\hline 16 & Solvent_distribution_H-0.75 & 0.601118 & 72 & Hydrophobicity_transition_NH & 0.680671 \\
\hline 17 & VanDerWaal_distribution_H-0.0 & 0.606629 & 73 & Hydrophobicity_distribution_H-0.75 & 0.679473 \\
\hline 18 & AA_composition_E & 0.612859 & 74 & Hydrophobicity_distribution_N-1.0 & 0.680511 \\
\hline 19 & AA_composition_R & 0.623802 & 75 & VanDerWaal_distribution_H-0.5 & 0.68099 \\
\hline 20 & AA_composition_F & 0.636422 & 76 & VanDerWaal_transition_PH & 0.679553 \\
\hline 21 & AA_composition_D & 0.641933 & 77 & Polarizability_composition_H & 0.678674 \\
\hline 22 & AA_composition_Y & 0.647843 & 78 & Polarizability_transition_NH & 0.678754 \\
\hline 23 & AA_composition_P & 0.653035 & 79 & Hydrophobicity_transition_PN & 0.677556 \\
\hline 24 & AA_composition_Q & 0.657987 & 80 & Polarizability_distribution_P-1.0 & 0.678594 \\
\hline 25 & Hydrophobicity_distribution_P-0.25 & 0.66238 & 81 & Hydrophobicity_distribution_H-0.5 & 0.678434 \\
\hline 26 & Hydrophobicity_transition_PH & 0.668291 & 82 & Polarizability_transition_PN & 0.678355 \\
\hline 27 & Hydrophobicity_distribution_P-1.0 & 0.671086 & 83 & Polarity_distribution_H-0.5 & 0.677077 \\
\hline 28 & AA_composition_N & 0.67492 & 84 & VanDerWaal_distribution_H-0.75 & 0.676198 \\
\hline 29 & Solvent_distribution_H-1.0 & 0.675399 & 85 & Solvent_distribution_H- $0 . \overline{5}$ & 0.675639 \\
\hline 30 & Hydrophobicity_composition_P & 0.675399 & 86 & VanDerWaal_distribution_P-0.25 & 0.676198 \\
\hline 31 & Polarity_transition_PN & 0.677955 & 87 & Polarizability_distribution_N-1.0 & 0.67516 \\
\hline 32 & Polarizability_transition_PH & 0.679792 & 88 & Polarizability_distribution_P-0.0 & 0.675399 \\
\hline 33 & Solvent_transition_HE & 0.680751 & 89 & VanDerWaal_distribution_P-0.5 & 0.67524 \\
\hline 34 & Polarity_distribution_H-0.75 & 0.68107 & 90 & Solvent_distribution_H-0. & 0.673642 \\
\hline 35 & Polarizability_distribution_P-0.5 & 0.684185 & 91 & Polarity_distribution_N-1.0 & 0.673083 \\
\hline 36 & AA_composition_H & 0.686262 & 92 & VanDerWaal_composition_H & 0.672204 \\
\hline 37 & Polarizability_distribution_H-0.0 & 0.688419 & 93 & Polarizability_distribution_H- 0.5 & 0.671166 \\
\hline 38 & VanDerWaal_distribution_N-1.0 & 0.688419 & 94 & Polarity_distribution_N-0.25 & 0.670367 \\
\hline 39 & Polarity_composition_N & 0.688339 & 95 & Hydrophobicity_distribution_H-0.0 & 0.670288 \\
\hline 40 & Hydrophobicity_distribution_P-0.75 & 0.688259 & 96 & Polarizability_distribution_N-0.0 & 0.669409 \\
\hline 41 & Polarity_distribution_P-0.75 & 0.688179 & 97 & Polarizability_distribution_N-0.25 & 0.669249 \\
\hline 42 & Hydrophobicity_distribution_P-0.0 & 0.686981 & 98 & VanDerWaal_distribution_H-1.0 & 0.66845 \\
\hline 43 & Hydrophobicity_distribution_P-0.5 & 0.686981 & 99 & VanDerWaal_distribution_N-0.75 & 0.667332 \\
\hline 44 & Polarity_distribution_H-0.0 & 0.685623 & 100 & Polarizability_composition_N & 0.666773 \\
\hline 45 & Polarizability_distribution_H-1.0 & 0.685863 & 101 & Polarizability_distribution_N- -0.75 & 0.665575 \\
\hline 46 & Polarity_transition_PH & 0.685783 & 102 & VanDerWaal_distribution_P-0.0 & 0.665895 \\
\hline 47 & Polarity_composition_P & 0.686661 & 103 & VanDerWaal_distribution_P-0.75 & 0.664697 \\
\hline 48 & Polarity_distribution_P-0.0 & 0.686422 & 104 & Polarizability_distribution_P-0.75 & 0.663259 \\
\hline 49 & Hydrophobicity_distribution_N-0.0 & 0.685942 & 105 & Polarity_distribution_N-0.0 & 0.661821 \\
\hline 50 & AA_composition_K & 0.686981 & 106 & Polarizability_distribution_H-0.75 & 0.659984 \\
\hline 51 & Polarity_distribution_N-0.75 & 0.686182 & 107 & Polarity_transition_NH & 0.657907 \\
\hline 52 & Polarity_distribution_N-0.5 & 0.687061 & 108 & Hydrophobicity_distribution_N-0.25 & 0.657029 \\
\hline 53 & VanDerWaal_distribution_N-0.0 & 0.686102 & 109 & VanDerWaal_distribution_P-1.0 & 0.65599 \\
\hline 54 & Hydrophobicity_composition_N & 0.686342 & 110 & VanDerWaal_distribution_H-0.25 & 0.655032 \\
\hline 55 & Polarizability_composition_P & 0.684585 & 111 & Polarizability_distribution_H- 0.25 & 0.654952 \\
\hline 56 & VanDerWaal_transition_PN & 0.684105 & & & \\
\hline
\end{tabular}

The first 18 features come from mRMR features. The highest accuracy is $68.8 \%$, which takes place when the 37 th feature is added in. Please refer to the footnote in Table 3 for the meaning of the feature names 
analysis. The 18 most relative features mainly consists of amino acid composition, solvent accessibility and normalized Van Der Waals volume, with the number being 9, 4 and 3 , respectively (see Table 5). There is no doubt that amino acid composition is vital for maintaining the function of proteins $[26,27]$. From Table 5, the most contributing features are amino acid compositions: in the first 5 features, 3 of them are amino acid compositions; in the first 10 features, 7 of them are amino acid compositions; in the first 15 features, 9 of them are amino acid compositions. Among the selected 37 features, 18 of them are amino acid compositions. In all cases, the amino acid compositions occupy about half or more than half of the features. Therefore, we conclude that the amino acid compositions contribute more to the prediction than the physiochemical features, and the hypothesis is false. As for solvent accessibility, it has been known that residues located in the surface of a protein usually function as active sites interacting with other molecules and ligands. For example, DNA-binding proteins prefer to bind with residues with higher solvent accessible area [28]; in the mutants of Hsc70 proteins, the reduction in solvent accessibility results a higher hydrophobic free energy level [29]. Thus solvent accessibility was widely used for drug design and fold recognition [30,31]. The solvent-accessible surface contributes to the stability of a ligand receptor complex [32], protein crystal stability [33] and was used to model side chain conforma-

Table 5 Distribution of the first 18 mRMR features in the first 22 MaxRel features

\begin{tabular}{lll}
\hline Order & Name & Score \\
\hline 1 & AA_composition_C & 0.267 \\
2 & Solventcomposition_H & 0.192 \\
3 & VanDerWaal_composition_N & 0.145 \\
4 & AA_composition_L & 0.138 \\
5 & VanDerWaal_composition_P & 0.125 \\
6 & Polarity_composition_H & 0.117 \\
7 & Hydrophobicity_composition_N & 0.102 \\
8 & AA_composition_T & 0.102 \\
9 & AA_composition_V & 0.101 \\
10 & Hydrophobicity_composition_P & 0.101 \\
11 & AA_composition_E & 0.088 \\
12 & Polarity_composition_N & 0.086 \\
13 & AA_composition_G & 0.085 \\
14 & VanDerWaal_transition_NH & 0.084 \\
15 & AA_composition_A & 0.081 \\
16 & AA_composition_S & 0.079 \\
17 & VanDerWaal_distribution_N-O.25 & 0.074 \\
18 & Solvent_distribution_H-O.75 & 0.073 \\
19 & Solvent_distribution_H-O.O & 0.072 \\
20 & AA_composition_W & 0.071 \\
21 & Polarity_distribution_P-0.0 & 0.070 \\
22 & Solvent_transition_HEE & 0.069 \\
\hline
\end{tabular}

The italicized ones are the mRMR features also appearing in the final selected 37 features. The first 18 mRMR features mainly consist of amino acid compositions, solvent accessibility and normalized Van Der Waal volume with the number being 9,4 and 3 , respectively tions [34]. And we also know that the solvent-accessible surface is derived from the normalized Van Der Waals volume [35]. Undoubtedly they are strongly related to each other, and together they help to predict the protein structural classes.

\section{Conclusions}

By combining the NNA, mRMR and feature forward searching strategy, we are able to investigate the role of physiochemical properties in predicting the protein structural classes. The results are summarized as followings. Physiochemical features do provide extra prediction capacity to the original amino acid compositions. However, the hypothesis that physiochemical properties provide more prediction capacity is proved to be false. Therefore, more researches should be carried out to search more profound information derived from amino acid sequences. Solvent accessibility and normalized Van Der Waals volume contribute more to the prediction of the protein structural classes than other physiochemical features in the prediction. The combination of mRMR and forward feature searching method provides a way to effectively and efficiently develop the predicting models on the large dataset with hundreds of or more features. A combination method shall be chosen as to achieve good balance between computation speed and the successfulness in the feature selection. Finally, the predictor developed in this study is available on: http://app3.biosino.org:8080/liwenjin/index. jsp

Acknowledgements The authors thank Ziliang Qian, a PhD student in SIBS of CAS, for his help to develop the web-server.

\section{References}

1. Chou KC, Zhang CT (1995) Prediction of protein structural classes. Crit Rev Biochem Mol Biol 30:275-349. doi:10.3109/ 10409239509083488

2. Chou PY, Fasman GD (1978) Prediction of the secondary structure of proteins from their amino acid sequence. Adv Enzymol Relat Areas Mol Biol 47:45-148

3. Klein P, Delisi C (1986) Prediction of protein structural class from the amino acid sequence. Biopolymers 25:1659-1672. doi:10. 1002/bip.360250909

4. Nakashima H, Nishikawa K, Ooi T (1986) The folding type of a protein is relevant to the amino acid composition. $\mathrm{J}$ Biochem 99:153-162

5. Zhang CT, Chou KC (1992) An optimization approach to predicting protein structural class from amino acid composition. Protein Sci 1:401-408

6. Chou KC, Maggiora GM (1998) Domain structural class prediction. Protein Eng 11:523-538. doi:10.1093/protein/11.7.523

7. Chou KC, Zhang CT (1992) A correlation-coefficient method to predicting protein-structural classes from amino acid compositions. Eur J Biochem 207:429-433. doi:10.1111/j.1432-1033. 1992.tb17067.x 
8. Cai YD, Feng KY, Lu WC, Chou KC (2006) Using LogitBoost classifier to predict protein structural classes. J Theor Biol 238:172176. doi:10.1016/j.jtbi.2005.05.034

9. Xiao X, Shao SH, Huang ZD, Chou KC (2006) Using pseudo amino acid composition to predict protein structural classes: approached with complexity measure factor. J Comput Chem 27:478-482. doi:10.1002/jec.20354

10. Dubchak I, Muchnik I, Holbrook SR, Kim SH (1995) Prediction of protein folding class using global description of amino acid sequence. Proc Natl Acad Sci USA 92:8700-8704. doi:10.1073/ pnas.92.19.8700

11. Wang ZX, Yuan Z (2000) How good is prediction of protein structural class by the component-coupled method? Proteins 38:165-175. doi:10.1002/(SICI)1097-0134(20000201)38:2<165:: AID-PROT5>3.0.CO;2-V

12. Chou KC, Cai YD (2004) Predicting protein structural class by functional domain composition. Biochem Biophys Res Commun 321:1007-1009. doi:10.1016/j.bbrc.2004.07.059

13. Cao Y, Liu S, Zhang L, Qin J, Wang J, Tang K (2006) Prediction of protein structural class with Rough Sets. BMC Bioinformatics 7:1-6. doi:10.1186/1471-2105-7-20

14. Ding YS, Zhang TL, Chou KC (2007) Prediction of protein structure classes with pseudo amino acid composition and fuzzy support vector machine network. Protein Pept Lett 14:811-815. doi:10. 2174/092986607781483778

15. Peng H, Long F, Ding C (2005) Feature selection based on mutual information: criteria of max-dependency, max-relevance, and minredundancy. IEEE Trans Pattern Anal Mach Intell 27:1226-1238. doi:10.1109/TPAMI.2005.159

16. Cai YD, Chou KC (2003) Nearest neighbour algorithm for predicting protein subcellular location by combining functional domain composition and pseudo-amino acid composition. Biochem Biophys Res Commun 305:407-411. doi:10.1016/ S0006-291X(03)00775-7

17. Murzin AG, Brenner SE, Hubbard T, Chothia C (1995) SCOP: a structural classification of proteins database for the investigation of sequences and structures. J Mol Biol 247:536-540

18. Lo Conte L, Brenner SE, Hubbard TJ, Chothia C, Murzin AG (2002) SCOP database in 2002: refinements accommodate structural genomics. Nucleic Acids Res 30:264-267. doi:10.1093/nar/ 30.1.264

19. Andreeva A, Howorth D, Brenner SE, Hubbard TJ, Chothia C, Murzin AG (2004) SCOP database in 2004: refinements integrate structure and sequence family data. Nucleic Acids Res 32:D226D229. doi:10.1093/nar/gkh039

20. Chandonia JM, Walker NS, Lo Conte L, Koehl P, Levitt M, Brenner SE (2002) ASTRAL compendium enhancements. Nucleic Acids Res 30:260-263. doi:10.1093/nar/30.1.260

21. Chandonia JM, Hon G, Walker NS, Lo Conte L, Koehl P, Levitt M, et al (2004) The ASTRAL Compendium in 2004. Nucleic Acids Res 32:D189-D192. doi:10.1093/nar/gkh034
22. Brenner SE, Koehl P, Levitt M (2000) The ASTRAL compendium for protein structure and sequence analysis. Nucleic Acids Res 28:254-256. doi:10.1093/nar/28.1.254

23. Dubchak I, Muchnik I, Mayor C, Dralyuk I, Kim SH (1999) Recognition of a protein fold in the context of the Structural Classification of Proteins (SCOP) classification. Proteins 35:401-407. doi:10.1002/(SICI)1097-0134(19990601)35:4<401:: AID-PROT3>3.0.CO;2-K

24. Mucchielli-Giorgi MH, Hazout S, Tuffery P (1999) PredAcc: prediction of solvent accessibility. Bioinformatics 15:176-177. doi:10.1093/bioinformatics/15.2.176

25. Ding C, Peng H (2005) Minimum redundancy feature selection from microarray gene expression data. J Bioinform Comput Biol 3:185-205. doi:10.1142/S0219720005001004

26. Weng Z, Rickles RJ, Feng S, Richard S, Shaw AS, Schreiber SL et al (1995) Structure-function analysis of SH3 domains: SH3 binding specificity altered by single amino acid substitutions. Mol Cell Biol 15:5627-5634

27. Hansen JC, Lu X, Ross ED, Woody RW (2006) Intrinsic protein disorder, amino acid composition, and histone terminal domains. J Biol Chem 281:1853-1856. doi:10.1074/jbc.R500022200

28. Ahmad S, Gromiha MM, Sarai A (2004) Analysis and prediction of DNA-binding proteins and their binding residues based on composition, sequence and structural information. Bioinformatics 20:477-486. doi:10.1093/bioinformatics/btg432

29. Kumarevel TS, Gromiha MM, Ponnuswamy MN (1998) Solvent accessibility analysis on the mutants of Hsc70 ATPase fragment. Biophys Chem 71:99-111. doi:10.1016/S0301-4622(97)00137-3

30. Gromiha MM, Ahmad S (2005) Role of solvent accessibility in structure based drug design. Curr Comput-Aided Drug Des 1:223235. doi:10.2174/1573409054367664

31. Liu S, Zhang C, Liang S, Zhou Y (2007) Fold recognition by concurrent use of solvent accessibility and residue depth. Proteins 68:636-645. doi:10.1002/prot.21459

32. Froeyen M, DeWinter H, Herdewijn P (2006) Conformational analysis, solvent-accessible surface and geometric extent of inhibitors and substrates. Collect Czech Chem Commun 71:842858. doi: $10.1135 / \operatorname{ccc} 20060842$

33. Islam SA, Weaver DL (1990) Molecular interactions in protein crystals: solvent accessible surface and stability. Proteins 8:1-5. doi: $10.1002 /$ prot. 340080103

34. Eyal E, Najmanovich R, McConkey BJ, Edelman M, Sobolev V (2004) Importance of solvent accessibility and contact surfaces in modeling side-chain conformations in proteins. J Comput Chem 25:712-724. doi:10.1002/jcc. 10420

35. Connolly ML (1996) Molecular surfaces: A review. Solvent Accessible Surfaces http://www.netsci.org/Science/Compchem/ feature $14 \mathrm{e} . \mathrm{html}$ 